Federal Reserve Bank of Minneapolis

Research Department

\title{
A Model of Regulated Private Bank-Note Issue*
}

\author{
Ricardo de O. Cavalcanti and Neil Wallace \\ Working Paper 581
}

April 1997

\begin{abstract}
A random-matching model (of money) is formulated in which there is complete public knowledge of the trading histories of a subset of the population, called banks, and no public knowledge of the trading histories of the complement of that subset, called nonbanks. Each person, whether a banker or a non banker, is assumed to have the technological capability to create indivisible, distinct and durable objects called notes. If outside money is indivisible and sufficiently scarce, then an optimal mechanism is shown to have note issue and destruction (redemption) by banks.
\end{abstract}

*Cavalcanti, Department of Economics, University of Miami and EPGE-Fundação Getúlio Vargas, e-mail: ricardoc @atlas.socsci.umn.edu; Wallace, Department of Economics, University of Miami and Federal Reserve Bank of Minneapolis, e-mail: nwallace@umiami.ir.miami.edu. This paper was prepared for presentation at the SEDC Meeting at Oxford, July 1997. The views expressed herein are those of the authors and not necessarily those of the Federal Reserve Bank of Minneapolis or the Federal Reserve System. 


\title{
A Model of Regulated Private Bank-Note Issue*
}

\author{
Ricardo De O. Cavalcanti ${ }^{\dagger} \quad$ Neil Wallace ${ }^{\ddagger}$
}

April 23, 1997

\begin{abstract}
A random-matching model (of money) is formulated in which there is complete public knowledge of the trading histories of a subset of the population, called banks, and no public knowledge of the trading histories of the complement of that subset, called nonbanks. Each person, whether a banker or a non banker, is assumed to have the technological capability to create indivisible, distinct and durable objects called notes. If outside money is indivisible and sufficiently scarce, then an optimal mechanism is shown to have note issue and destruction (redemption) by banks.
\end{abstract}

\section{INTRODUCTION}

Shortages of media of exchange associated with the indivisibility and scarcity of commodity outside money were ubiquitous at least until the middle of the 19th century. One common response or proposed response was private, but regulated issue of bank notes. ${ }^{1}$ Such notes were issued in France and Scotland in the 18th century, in some of England's colonies and in the U.S. in the 19th century, and, no doubt, elsewhere and earlier. In this paper, we build on recent work on random-matching models of money and provide a model in which an optimal mechanism resembles regulated private bank-note issue when outside money is indivisible and sufficiently scarce.

Our model is closely related to Kocherlakota and Wallace (1997), but is also inspired by the model of banking in Cavalcanti et. al. (1997). Kocherlakota and Wallace (1997) generalize a random matching model of outside money by abandoning the assumption, implicit or explicit in most such models, that each person's trading

\footnotetext{
${ }^{*}$ This paper was prepared for presentation at the SEDC Meeting at Oxford, July 1997.

${ }^{\dagger}$ Department of Economics, University of Miami and EPGE - Fundação Getúlio Vargas. email<ricardoc@atlas.socsci.umn.edu $>$.

${ }^{\ddagger}$ Department of Economics, University of Miami and Federal Reserve Bank of Minneapolis. email<nwallace@umiami.ir.miami.edu>.

${ }^{1}$ An early proponent of note issue was John Law (1705). For a discussion of currency indivisibility in early modern Europe, see Glassman and Redish (1988). For a discussion of indivisibility and the role of banknotes in the American colonies, see Hanson (1979). For a discussion of early colonial Australia, see Butlin (1968).
} 
history is private information. They study a setting with partial public knowledge of individual trading histories, one in which the public record of everyone's trading history is updated with a lag. They show that if the lag is finite and sufficiently large, then the optimal mechanism has a role for transfers of resources in exchange for outside money and for other transfers of resources which can be thought of as credit, mutual charity, or insurance.

Here we adopt a different version of partial public knowledge of individual trading histories. We assume that there is complete public knowledge of the trading histories of a subset of the population, called the banking sector, and no public knowledge of the trading histories of the complement of that subset, called the non bank sector. The maximum size of the banking sector is a given feature of the technology. In other words, the society has a limited capacity to monitor trading histories; a given size subset of the population can be monitored perfectly and costlessly and no one outside that subset can be monitored at all. We also give each person, whether a banker or a non banker, the technological capability to create indivisible and durable objects called notes. We assume that the notes issued by a single person are uniform and distinct from those issued by any one else - distinct in terms of some observable but otherwise irrelevant characteristic like color or identifying number. We also assume that any person can always destroy or freely dispose of notes. When issued by bankers, the notes are the model's analog of the banknotes issued by private banks in the 18th and 19th centuries. A complete description of the environment we study appears in section 2 .

In section 3, we study a special case with no banking sector and with an exogenous amount of indivisible outside money. This special case serves at least two purposes. First, because it is very simple, it serves as an introduction to the specification and study of the more complicated setting with a banking sector. Second, several results for this special case anticipate results in the setting with a banking sector. For the special case, there is no role for notes issued by non bankers and ex ante welfare depends in a simple way on the amount of outside money.

In section 4, we assume that there is no outside money, but that there is a positive measure of people whose histories are public information, our banking sector. In order to be able to interpret banking as private, we maximize the steady state expected utility of non bankers subject to making bankers at least as well off as non bankers. We show that any optimum has positive note issue by bankers and has bankers destroying (redeeming) bank notes in a way that is interpretable as resulting from regulation. And for a banking sector that is sufficiently small, we are able to further describe the optimum. Welfare depends on the amount of notes issued by bankers and held by non bankers, a form of inside money, in a way that resembles its dependence on the amount of outside money in section 3, and welfare is increasing in the size of the banking sector. By continuity, those results hold when the amount of indivisible 
outside money is sufficiently small.

We conclude with a discussion of our assumptions. In particular, we discuss the senses in which banks in the model differ from historically observed note-issuing banks. We suggest that the most significant difference arises because we assume perfect and costless monitoring of banks rather than the imperfect and costly monitoring that actually existed.

\section{THE ENVIRONMENT}

Time is discrete and the horizon is infinite. There are $N$ distinct divisible and perishable types of goods at each date and there is a $[0,1]$ continuum of each of $N$ types of people, where $N \geq 3$. Each type is specialized in consumption and production: a type $n$ person consumes good $n$ and produces good $n+1(\operatorname{modulo} N)$, for $n=1,2, \ldots, N$. Each type $n$ person maximizes expected discounted utility with discount factor $\beta \in(0,1)$. Utility in a period is given by $u(x)-y$, where $x$ is the amount consumed and $y$ is the amount produced (see figure 1). The function $u$ is defined on $[0, \infty)$, is increasing, twice differentiable and bounded, and satisfies $u(0)=0, u^{\prime \prime}<0$, and $u^{\prime}(0)=\infty .^{2}$

In each period, people are randomly matched in pairs. Meetings are of two sorts: single-coincidence meetings, those between a type $n$ person (the producer) and a type $n+1$ person (the consumer) for some $n$; and no-coincidence meetings, those in which neither person produces what the other consumes. (Because the number of types, $N$, exceeds two, there are no double-coincidence meetings.) We assume that people in a meeting know each other's type. We also assume that people cannot precommit to future actions, so that those who produce in a single-coincidence meeting have to get a future reward for doing so. ${ }^{3}$

The society is able to keep a public record of a fraction $b N$ of each type of person. It has no public record of the complement of $b N$. If $b$ is equal to $\frac{1}{N}$, then there is a complete public record of every person's actions. If $b$ is equal to zero, then there is no public record of any person's actions. For both of those special cases, the model reduces to special cases of Kocherlakota-Wallace (1997). As we will see, a person whose history is known can be induced to produce without receiving something tangible in exchange, because the person can be rewarded and punished in the future

\footnotetext{
${ }^{2}$ Boundedness of $u$ can be interpreted as coming from a bound on production. Suppose that the utility of consuming is $r$, which satisfies all the assumptions made about $u$ except boundedness, and suppose that $d$ is the disutility of production, where $d:[0, Z) \rightarrow \mathbb{R}_{+}$is strictly convex, with $d(z)$ and $d^{\prime}(z) \rightarrow \infty$ as $z \rightarrow Z$. This is equivalent to our specification if we let $x \equiv d(z)$ and let $u(x) \equiv r\left[d^{-1}(x)\right]$.

${ }^{3}$ The above specification is much like that in Shi (1995) and Trejos and Wright (1995).
} 
for actions they take currently; in contrast, a person whose history is not known must receive something tangible in order to produce.

We also assume that each person has a technology that permits the person to create indivisible and perfectly durable objects. The objects, called notes, issued by a single person are uniform, but distinguishable from those issued by any one else, so that counterfeiting is not a problem. We also assume that any person can always destroy or freely dispose of notes that the person has acquired. We will see that an optimum does not require note issue by those whose histories are private information, the non bankers, and does require note issue and destruction (redemption) by those whose histories are known, the bankers.

To keep the model simple, we assume that each person can carry from one date to the next at most one unit of notes issued by others or one unit of outside money. As we explain below, we believe that our main results are robust to weakening that assumption. We also assume that each trader in a meeting is able to see the trading partner's note or outside money holdings.

\section{OUtSide MONEY AND NO BANKING SECTOR}

Here we assume that $b=0$ and that there is some outside money. We assume a symmetric initial distribution of outside money and, therefore, let $m_{i}$ denote the fraction of each type with $i$ units of money divided by $N, i \in\{0,1\}$, so that $m_{0}+m_{1}=$ $\frac{1}{N}$. We also assume that $m_{0}$ is positive so that not everyone is at capacity when all the outside money is held. We begin by specifying the set of incentive feasible allocations and do this exactly as in Kocherlakota-Wallace (1997). We first describe a trading mechanism in which people in meetings play a coordination game: two people in a meeting simultaneously propose a net trade; if they name the same net trade, then that trade occurs; if they name different net trades, then each person leaves the meeting and proceeds to the next date with what the person owned prior to the meeting. Then we define symmetric and stationary equilibrium outcomes of this mechanism. ${ }^{4}$ An allocation is incentive feasible if it is one of these equilibria.

To simplify the exposition, we anticipate some of the consequences of symmetry in our description of the strategies in meetings. One symmetry we impose is that all notes issued by non bankers are treated the same. This implies that a non banker will never produce in order to acquire a note issued by a non banker because the non banker could always hand out his or her own note instead. In other words, such symmetry assures that notes issued by non bankers do not circulate among non

\footnotetext{
${ }^{4}$ Kocherlakota and Wallace show that the set of symmetric and stationary equilibrium outcomes of this coordination mechanism is the same as those of a somewhat general trading mechanism that permits each person to leave a meeting without trading. Their first proposition applies to this setting.
} 
bankers. It follows that such notes are superfluous. ${ }^{5}$ We use that fact and ignore note issue by non bankers in what follows. We also anticipate, as we will explain, some of the incentive constraints.

Meetings are either single-coincidence meetings or no-coincidence meetings. When two non bankers meet, trade can occur only if the meeting is a single-coincidence meeting and if the producer does not have money and the consumer does - a consequence of the fact that trade among non bankers must be quid pro quo trade. In such a meeting, each non banker names a level of production, $x_{n}$, to be produced by the producer in the meeting and consumed by the consumer in the meeting. If they name the same positive amount, then they behave accordingly and the consumer transfers his or her unit of money to the producer; otherwise, nothing happens in the meeting and they go on to the next date.

We denote by $s$ coordinated actions in the above game that are symmetric across agent types and stationary. Symmetry and stationarity include the requirement that the action taken in a meeting depends only on the kind of meeting the people are in. Thus, $s$ is simply some $x_{n} \in \mathbb{R}_{+}$, and $s$ is incentive feasible if no one wants to deviate from the $x_{n}$ specified. ${ }^{6}$

In order to express the no-deviation conditions, we first describe in terms of $x_{n}$ the expected discounted utilities of non bankers as of the beginning of a period before a meeting. We let $v_{i}$ be that of a non banker with $i$ units of money. For $x_{n}>0$, we have

$$
v_{1}=\beta v_{1}+m_{0}\left[u\left(x_{n}\right)+\beta\left(v_{0}-v_{1}\right)\right]
$$

and

$$
v_{0}=\beta v_{0}+m_{1}\left[-x_{n}+\beta\left(v_{1}-v_{0}\right)\right] .
$$

These expressions are written under the presumption that when $x_{n}$ is produced and consumed, a unit of outside money changes hands. Notice that $m_{i}$ is the probability of meeting a particular type with $i$ units of money. In the expression for $v_{1}, m_{0}$ is the probability of meeting a producer without money, while in the expression for $v_{0}$, $m_{1}$ is the probability of meeting a consumer with a unit of money. The no-deviation conditions for non bankers assume that defection on the part of non bankers goes unpunished because such defection does not become part of a public record. Thus, the conditions are simply that the action in $s$ is weakly preferred to leaving the

\footnotetext{
${ }^{5}$ Even without symmetry, note issue by a subset of non bankers is not consistent with a steady state because no one would retire or destroy such notes and no individual banker in the subset issuing notes would refrain from issuing another.

${ }^{6}$ Kocherlakota and Wallace include in $s$ production levels by the consumer and production levels in no-coincidence meetings and show that those should be zero in the case under consideration.
} 
meeting with what was brought into the meeting. There are two such conditions, one for the consumer and one for the producer:

$$
u\left(x_{n}\right)+\beta\left(v_{0}-v_{1}\right) \geq 0
$$

and

$$
-x_{n}+\beta\left(v_{1}-v_{0}\right) \geq 0 .
$$

These conditions, non negative gains from trade, give rise to the following definition of incentive feasible allocations.

Definition 1. An allocation $s=x_{n}$ is incentive feasible if there exists $\left(v_{1}, v_{0}\right)$ such that (1)-(4) hold.

Let $v=N\left(m_{1} v_{1}+m_{0} v_{0}\right)$ define the expected utility of non bankers, an expectation taken with respect to the stationary (and initial) distribution of money holdings for non bankers. Then our optimum problem is the following.

Problem 1. Maximize $v$ by choice of a feasible $s$ (an $x_{n}$ that satisfies definition 1$)$.

This problem is easily solved. By (1) and (2),

$$
\frac{1-\beta}{N} v=m_{0} m_{1}\left[u\left(x_{n}\right)-x_{n}\right]
$$

and

$$
\left(\rho+\frac{1}{N}\right) \beta\left(v_{1}-v_{0}\right)=m_{0} u\left(x_{n}\right)+m_{1} x_{n},
$$

where $\rho=(1-\beta) / \beta$. Using (6), inequality (4) is equivalent to

$$
\left(1+\frac{\rho}{m_{0}}\right) x_{n} \leq u\left(x_{n}\right)
$$

while inequality (3) is equivalent to $\left[m_{1} /\left(m_{1}+\rho\right)\right] x_{n} \leq u\left(x_{n}\right)$. It follows that satisfaction of (7) implies satisfaction of (3). Therefore, by (5), problem 1 is equivalent to choosing $x_{n}$ to maximize $u\left(x_{n}\right)-x_{n}$ subject to $(7)$. Let $x^{\prime}$ be the unique solution to $\frac{d u(x)}{d x}=1$ and let $x^{\prime \prime}$ be the unique positive $x_{n}$ satisfying (7) at equality. Then the solution to problem 1 is $x_{n}=x^{*}$, where $x^{*}=\min \left\{x^{\prime}, x^{\prime \prime}\right\}$ (see figure 1). Because $m_{0}>0, x^{*}>0$.

We now describe how the solution depends on the amount of money, $m_{1}$. First, the constraint set, (7), is decreasing in the amount of money. And because $m_{0} m_{1}$ is a maximum at $m_{0}=m_{1}=\frac{1}{2 N}$, it follows that welfare is strictly decreasing in the 


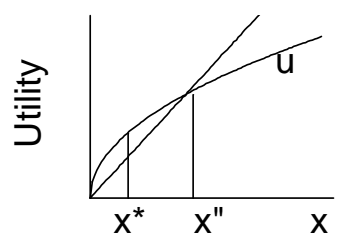

(i) $\mathrm{x}^{*}=\mathrm{x}^{\prime}$

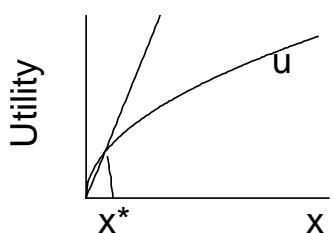

(ii) $\mathrm{X}^{*}=\mathrm{x}^{\prime \prime}$

Figure 1: Possible solutions with no banking sector

amount of money for $m_{1} \geq \frac{1}{2 N}$. For $m_{1} \leq \frac{1}{2 N}$, welfare depends on whether constraint (7) is binding. If $x^{*}<x^{\prime}$ at $m_{1}=\frac{1}{2 N}$, then welfare reaches a maximum at some $m_{1} \in\left(0, \frac{1}{2 N}\right)$; otherwise, it reaches a maximum at $m_{1}=\frac{1}{2 N}$. Notice that the price of the good in terms of money, which is also the price level in this economy, is $\frac{1}{x^{*}}$. If there is some $m_{0}<\frac{1}{N}$, say $m_{0}^{\prime}$, such that $x^{\prime}$ satisfies $(7)$ with equality at $m_{0}=m_{0}^{\prime}$, then the solution to problem 1 is $x_{n}=x^{\prime}$ for all $m_{1} \leq \frac{1}{N}-m_{0}^{\prime}$. Therefore, for all such low amounts of money, the solution is a price that does not depend on the amount of money. ${ }^{7}$ For all $m_{1} \geq \frac{1}{N}-m_{0}^{\prime}$, the solution is a price that is increasing in the amount of money. Alternatively, in terms of bargaining or the division of the gains from trade, for all $m_{1}<\frac{1}{N}-m_{0}^{\prime}$, the gains are divided among the consumer and the producer with more going to the consumer the larger is $m_{1}$. For all $m_{1} \geq \frac{1}{N}-m_{0}^{\prime}$, all the gains go to the consumer and $v_{0}=0$.

As just noted, we can interpret the solution to problem 1 as a suggested price. In the coordination game, a potential defector sees as options either trading at the suggested price or not trading at all. However, the solution to problem 1 is not only Nash given those options; it is also robust to cooperative deviation by a pair in a meeting. Because the game in a meeting resembles a fixed-sum game (the producer would like to give up as little as possible for a unit of money and the consumer would like to get as much as possible for a unit of money), there is no cooperative and favorable deviation from the problem 1 solution for a pair in a meeting.

\section{A BANKING SECTOR AND NO OUTSIDE MONEY}

We now assume that there is a banking sector; that is, $b \in\left(0, \frac{1}{N}\right]$. We are interpreting $b$ as both the exogenous maximum size of the banking sector and the actual size. For $b$ sufficiently close to zero, which we consider the relevant assumption historically, proposition 3 implies that full utilization of the capacity of the banking sector is optimal. And to keep matters relatively simple, we now assume that there is no outside

\footnotetext{
${ }^{7}$ In this case, the solution resembles a customary price that over a range does not depend on the amount of money.
} 
money. We again follow Kocherlakota and Wallace in defining incentive feasible allocations. We first describe the appropriate coordination game in which two people in a meeting simultaneously propose a net trade. As above, the net trade is carried out if they propose the same net trade; otherwise each leaves the meeting with what the person brought into the meeting. Then we define symmetric and stationary equilibrium outcomes of this game. ${ }^{8}$ An allocation is incentive feasible if it is one of these equilibria. Symmetry and stationarity have important consequences here.

As in section 3, we anticipate some of the consequences of symmetry in our description of the strategies in meetings. We now assume that all notes issued by non bankers are treated the same and that all notes issued by (nondefecting) bankers are treated the same. The first implies, as above, that a non banker does not produce in order to acquire a note issued by a non banker. It follows, as above, that such notes are superfluous. ${ }^{9}$ In particular, they are not needed to induce bankers to produce, because the trading histories of bankers are known. Therefore, we continue to ignore note issue by non bankers and, henceforth, use the term notes to refer to notes issued by bankers. The assumption that all notes issued by bankers are treated the same implies that they are superfluous in trades between bankers. We also anticipate, as we will explain, some of the incentive constraints.

We describe, in turn, meetings between two bankers, between two non bankers, and between a banker and a non banker. Meetings are either single-coincidence meetings or no-coincidence meetings. When two bankers meet, trade occurs only if the meeting is a single-coincidence meeting. In such a meeting, each names a level of production, $y$, to be produced by the producer in the meeting and consumed by the consumer in the meeting. When two non bankers meet, trade can occur only if the meeting is a single-coincidence meeting and if the producer does not have a note and the consumer does. In such a meeting, each names a level of production, $x_{n}$, to be produced by the producer in the meeting and consumed by the consumer in the meeting. If they name the same positive amount, then they behave accordingly and the consumer transfers his or her note to the producer; otherwise, nothing happens in the meeting and they go on to the next date.

There are four nonvacuous kinds of meetings between a banker and a non banker. If a non banker has a note, then the non banker cannot be induced to produce. Therefore, it is only necessary to consider a meeting between such a non banker who

\footnotetext{
${ }^{8}$ The Kocherlakota-Wallace demonstration that the set of symmetric and stationary equilibrium outcomes of this coordination mechanism is the same as those of a somewhat general trading mechanism that permits each person to leave a meeting without trading can be adapted to the setting in this section.

${ }^{9}$ The symmetry is crucial for this result. Without it, the following possibility arises: a subset of nonbankers issues notes which are used by the other non bankers and are retired by bankers. We have not studied whether such non banker note issue can be optimal.
} 
is a consumer in a single-coincidence meeting with a banker. They each name a level of production, $y_{1}$, and a note transfer variable $\delta \in\{0,1\}$, where 1 (0) means that the non banker surrenders (does not surrender) his or her note. If a non banker has no note, then there are three kinds of meetings to consider. If the meeting is a no-coincidence meeting, then each names a note transfer variable $\gamma \in\{0,1\}$. If the banker is the producer in a single-coincidence meeting, then each names a level of production, $y_{0}$, and a note transfer variable $\theta \in\{0,1\}$. Finally, if the non banker is the producer, they each name a level of production, $x_{b}$, and a note transfer variable $\alpha \in\{0,1\}$. For $\gamma, \theta$, and $\alpha, 1(0)$ means that the banker gives (does not give) the non banker a note.

We now denote by $s$ coordinated actions in the above game that are symmetric across agent types and stationary. Symmetry and stationarity include the requirement that the action taken in a meeting depends only on which of the above six kinds of meetings the people are in. Thus, $s$ is now the vector, $\left(x_{n}, x_{b}, y, y_{0}, y_{1}, \alpha, \gamma, \delta, \theta\right) \in \mathbb{R}_{+}^{9}$, where, as indicated above, the first two components are production levels by non bankers, the next three are production levels by bankers, and the remaining four variables, each an element of $\{0,1\}$, describe transfers of notes. A vector $s$ is an incentive feasible allocation if two conditions are satisfied: $s$ is consistent with an unchanging stock of notes held by non bankers and that stock and $s$ are such that no one wants to deviate from the actions in $s$.

In order to express the constant stock-of-notes condition, we now let $m_{i} N$ denote the fraction of each type who are non bankers and who start with $i$ notes $(i=0,1)$ and let $b N$ denote the fraction of each type who are bankers, so that

$$
m_{0}+m_{1}+b=\frac{1}{N} .
$$

In terms of this notation, $\left(m_{0}, m_{1}\right)$ is constant if $(8)$ holds and if

$$
m_{0} b \alpha+m_{0} b(N-2) \gamma+m_{0} b \theta=m_{1} b \delta
$$

where the left side of (9) is the inflow into note holdings of non bankers and the right side is the outflow. If $\alpha=\gamma=\theta=\delta=0$ (bankers neither issue notes nor accept them), then any $\left(m_{0}, m_{1}\right)$ satisfying (8) also satisfies (9). However, if $\alpha=\gamma=\theta=\delta=0$, then no stock of outstanding notes can be approached from nearby stocks. Because it makes sense to require that any positive stock be approachable from nearby stocks, we associate $\alpha=\gamma=\theta=\delta=0$ uniquely with $m_{1}=0$. Therefore, we add as a requirement,

$$
m_{1}>0 \text { implies }(\alpha, \gamma, \theta, \delta) \neq(0,0,0,0) .
$$

It follows that there are only seven possible steady state magnitudes of $m_{1}: m_{1}=0$, 
$m_{1}=m_{0}, m_{1}=2 m_{0}, m_{1}=(N-2) m_{0}, m_{1}=(N-1) m_{0}, m_{1}=N m_{0}$, and $m_{1}=$ $\frac{1}{N}-b{ }^{10}$ All except $m_{1}=0$ satisfy $m_{1} \geq m_{0}$.

In order to express the no-deviation conditions, we first describe in terms of the components of $s$ the expected discounted utilities of non bankers and that of bankers, each as of the beginning of a period before a meeting. We now let $v_{i}$ be that of a non banker with $i$ notes and let $w$ be that of a banker. We have

$$
\begin{aligned}
v_{1}= & \beta v_{1}+m_{0}\left[u\left(x_{n}\right)+\beta\left(v_{0}-v_{1}\right)\right]+b\left[u\left(y_{1}\right)+\delta \beta\left(v_{0}-v_{1}\right)\right], \\
v_{0}= & \beta v_{0}+m_{1}\left[-x_{n}+\beta\left(v_{1}-v_{0}\right)\right]+b\left[u\left(y_{0}\right)+\theta \beta\left(v_{1}-v_{0}\right)\right]+ \\
& b \alpha\left[-x_{b}+\beta\left(v_{1}-v_{0}\right)\right]+b(N-2) \gamma \beta\left(v_{1}-v_{0}\right)
\end{aligned}
$$

and

$$
w=\beta w+b[u(y)-y]+m_{0} \alpha u\left(x_{b}\right)-m_{0} y_{0}-m_{1} y_{1} .
$$

The expressions for $v_{1}$ and $v_{0}$ are written as a sum of the product of the probability of a particular kind of meeting and the net gain from that meeting implied by $s$. For example, the expression for $v_{1}$ uses the fact that a non banker with a note does not produce. With probability $m_{0}$, such a non banker is in a single-coincidence meeting with a non-banker producer who does not have a note, while with probability $b$ such a non banker is in a single-coincidence meeting with a banker producer. In the former meeting, the non banker surrenders his or her note, while in the latter meeting the non banker does if $\delta=1$. The expression for $v_{0}$ is analogous. The expression for $w$ reflects the fact that there is no individual state variable for bankers.

The following no-deviation conditions assume again that defection on the part of non bankers goes unpunished. In contrast, defection by a banker is punished by having the defecting bank get the payoff from autarky, which is zero. (Below, we offer two interpretations of how the autarky punishment of a defecting bank is accomplished.) The no-deviation conditions are now

$$
\begin{gathered}
u\left(x_{n}\right)+\beta\left(v_{0}-v_{1}\right) \geq 0, \\
u\left(y_{1}\right)+\beta\left(v_{0}-v_{1}\right) \geq 0 \text { if } \delta>0, \\
-x_{n}+\beta\left(v_{1}-v_{0}\right) \geq 0,
\end{gathered}
$$

\footnotetext{
${ }^{10}$ Note that $m_{0}=0$ is consistent with $\delta=0$ and $\max \{\alpha, \gamma, \theta\}=1$. In proposition 2 , we show that $m_{0}=0$ is not optimal.
} 


$$
\begin{gathered}
-x_{b}+\beta\left(v_{1}-v_{0}\right) \geq 0 \text { if } \alpha>0, \\
v_{1} \geq v_{0} \geq 0 \text { and } w \geq 0,
\end{gathered}
$$

and

$$
-\max \left\{y, y_{0}, y_{1}\right\}+\beta w \geq 0 .
$$

Inequalities (14)-(17) express the requirement that a non banker receive nonnegative gains from trade. Inequalities (18) express the free disposal possibility and the fact that autarky is always an option for any person. Inequality (19) expresses the requirement that the payoff to bankers when they produce cannot be worse than autarky.

These conditions give rise to the following definition of incentive feasible allocations.

Definition 2. An allocation $s=\left(x_{n}, x_{b}, y, y_{0}, y_{1}, \alpha, \gamma, \delta, \theta\right)$ is incentive feasible if there exists $\left(m_{0}, m_{1}\right)$ and $\left(v_{1}, v_{0}, w\right)$ such that (8)-(19) hold.

Our specification is consistent with two interpretations of how the autarky punishment of a defecting bank is accomplished. One interpretation is that the response to defection by a banker triggers autarkic play by every person in every subsequent meeting, $s \equiv 0$. The other is that it triggers autarkic play only by the defecting banker and by all trading partners of the defecting banker. The virtue of the first, relative to the second, is that it is robust to cooperative deviations by any pair in a meeting. If it is believed that $s \equiv 0$ will be played by everyone in the future, then there is no beneficial cooperative deviation by any pair in a meeting. The second interpretation is not robust to cooperative deviations by pairs in a meeting. The critical meeting is between a non banker without a note who produces what the defecting bank consumes. The non banker is not willing to deviate by accepting a note issued by the defecting bank; the note is identifiable and if it will not be accepted by others in the future, then the non banker does not produce currently to obtain it. However, the non banker is willing to deviate by accepting from the defecting bank a note issued by another bank, because, under the second alternative, such a note will be accepted by others in the future.

With $v=\left(m_{1} v_{1}+m_{0} v_{0}\right) /\left(m_{1}+m_{0}\right)$, the expected utility of non bankers, an expectation taken with respect to the stationary distribution of note holdings for non bankers, our optimum problem is the following.

Problem 2. Maximize $v$ subject to $w \geq v$ by choice of an incentive feasible $s$ (one that satisfies definition 2). 
The inequality $w \geq v$ assures that people are happy to be bankers, and, therefore, justifies labeling bankers as private. Our first result is that a solution to problem 2 exists and is continuous.

Proposition 1. A solution to problem 2 exists. If $v^{*}(b)$ denotes the maximized value of $v$ in problem 2 and if $G(b)$ denotes the set of maximizers, then $v^{*}(b)$ is a continuous function and $G$ is $u h c$ in $b$.

The proof (see the appendix) is an application of the Theorem of the Maximum. The boundedness of $u$ implies that the $v_{i}$ 's and $w$ are bounded and those bounds and the incentive constraints involving the production components of $s$ imply bounds on production. That fact is used in the proof of proposition 1. The fact that those bounds do not depend on $b$ is used in the proof of proposition 3 .

We now turn to some description of the solution to problem 2.

Proposition 2. If $b \in\left(0, \frac{1}{N}\right)$, then any solution to problem 2 has bankers both creating and destroying notes.

The proof (see the appendix) shows that any allocation without notes being created and destroyed is not optimal. Creation means $\max \{\alpha, \gamma, \theta\}=1$ and destruction means $\delta=1$. If both do not occur, then, by (8)-(10), either $m_{1}=0$ and $\max \{\alpha, \gamma, \theta\}=0$ (no non banker has a note and none are being created), or $m_{0}=0$ and $\delta=0$ (every non banker has a note and none are being destroyed). In either case, non bankers do not produce so that $v$ and $w$ depend only on the $y$ 's; in particular, non banker welfare depends only on $y_{0}$ and $y_{1}$, which in this case can be thought of as gifts from bankers. Starting with such a gift-only incentive feasible allocation, we construct an incentive feasible alternative with $m_{1} \in\left(0, \frac{1}{N}-b\right)$ and show that it increases both $v$ and $w$. The alternative we construct has $m_{1}=m_{0}$ and $\alpha=1$, note issue only in exchange for production.

Proposition 2 says that any optimum has note issue and destruction $(\delta=1)$ destruction in the sense that whenever a banker meets a non banker who has a note and who consumes what the banker produces, the banker produces enough to get the non banker to surrender the note. It is reasonable to interpret such action to be the result of regulation, because a banker who did not face punishment for deviating would not produce in order to acquire an outstanding note. Also, proposition 2 does not depend on the particular criterion we use. It also holds, as does proposition 1, for any welfare function that is increasing and continuous in $v$ and $w$.

We next turn to further description of the optimum, but for $b$ in a neighborhood of zero.

Proposition 3. Let $s^{*}(b)$ be a solution to problem 2 and let $v^{*}(b)$ be the maximized objective in that problem. If $b$ is sufficiently close to zero, then 
(i) $s^{*}(b)$ satisfies $\alpha=\delta=1$ and $\gamma=\theta=0$ (which implies $m_{0}=m_{1} \equiv m$ );

(ii) if $x^{\prime}$ denotes the solution to $\frac{d u(x)}{d x}=1$, if $x^{\prime \prime \prime}$ denotes the unique positive $x$ satisfying $\left(1+\frac{\rho}{m}\right) x=u(x)$, and if $\hat{x}=\min \left\{x^{\prime}, x^{\prime \prime \prime}\right\}$, then $v^{*}(b) \rightarrow \frac{m}{2(1-\beta)}[u(\hat{x})-\hat{x}]$ as $b \rightarrow 0$; and

(iii) $v^{*}(b)$ is strictly increasing in $b$.

The proof (see the appendix) uses the fact that if $b$ sufficiently close to 0 , then the main determinant of $v$ is what happens in trades between two non bankers. In accord with what we saw in section 3 , those trades are more beneficial with $m_{0}=m_{1}$ than with $m_{1}$ discreetly higher than $m_{0}$, the only alternative other than $m_{1}=0$. We first show that $m_{1}=m_{0}$ is necessary for a maximum of $v$ without regard to the constraint $w \geq v$. We then complete the proof of the first part of the proposition by showing that $\alpha=1$ is necessary and sufficient for $w \geq v$. Necessity follows because with $b$ close to zero, production by non bankers is the only source of positive utility for bankers. Sufficiency follows from choosing an allocation with $y_{0}=0$ and $y_{1}=x_{b}=x_{n}=y=x$ for an appropriately chosen $x$, one that approaches $\hat{x}$ as $b \rightarrow 0$. (Notice that $\hat{x}$ is the optimal $x_{n}$ in section 3 when the amount of outside money satisfies $m_{1}=m_{0}$.) This choice makes $w>v$, because whether a banker produces and consumes in a meeting with a non banker depends only on the note holdings of the non banker, while whether a non banker produces and consumes in a meeting with a non banker depends on the note holdings of both of them. The proofs of the remaining parts of proposition 3 follow easily.

In the proof of proposition 3 , we produce an explicit incentive feasible allocation by maximizing $v$ by choice of $x$ for allocations with $\delta=\alpha=1, \gamma=\theta=0, y_{0}=0$, and $y_{1}=x_{b}=x_{n}=y=x$. The resulting $x$ depends on $b$ and we denote the implied allocation by $s(b)$ and the implied $v$ and $w$ by $v[s(b)]$ and $w[s(b)]$, respectively. In accord with the explanation above, $w[s(b)]>v[s(b)]$ and both are strictly increasing in $b$. The function $v^{*}(b)$ is continuous (proposition 1 ) on $\left(0, \frac{1}{N}\right]$, is bounded below by $v[s(b)]$ and has $v[s(b)]$ as a limit as $b \rightarrow 0$ (see proposition 3). Also, $v^{*}\left(\frac{1}{N}\right)=w\left[s\left(\frac{1}{N}\right)\right]$, because as $b \rightarrow \frac{1}{N}$, both $v$ and $w$ are determined by what happens in meetings with bankers and nothing prevents bankers from treating non bankers as they treat bankers. These facts about $v^{*}(b)$ are depicted in figure 2. We have not been able to show that $v^{*}(b)$ is bounded above by $w[s(b)]$ or that it is increasing throughout its domain.

The optimum we find is discontinuous in $b$ at $b=0$ in the sense that $b=0$ implies autarky $(v=0)$, while $b>0$ implies a lower bound on $v$ given by $v[s(b)]$, which is positive and approaches a positive limit as $b \rightarrow 0$. However, that discontinuity at $b=0$ is an artifact of restricting attention to steady states. For an initial condition with no banknotes outstanding, the only one that is consistent with $b=0$, welfare would 


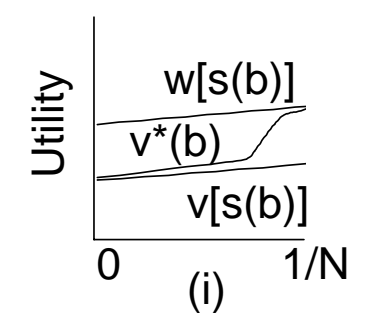

(i)

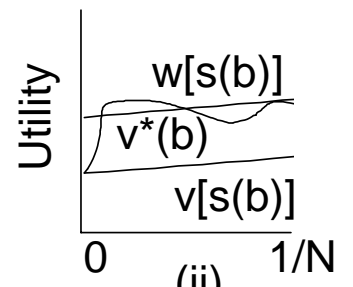

(ii)

Figure 2: Possible solutions with a banking sector

be continuous in $b$ at $b=0$, because a sufficiently small $b$ under any policy implies a sufficiently slowly growing stock of notes in use among non bankers. Therefore, ex ante welfare of non bankers would approach zero as $b \rightarrow 0$.

\section{CONCLUding REMARKS}

Because note issue was fairly widespread and because it was often suggested as a remedy for currency shortages even when it did not occur, it ought to be rationalized easily. We have shown that three historically plausible assumptions make it optimal. First, outside money is indivisible and scarce. Second, a tangible medium of exchange is essential because of a lack of double coincidences, an absence of commitment, and some privacy of individual trading histories. Third, the privacy of individual trading histories is not uniform over people; more is known or can be learned about the trading histories of some people than others. In particular, our model implies that those whose trading histories are known should issue notes, a kind of inside money, that can be used in trade among those whose trading histories are not known. As we noted, that result holds for our welfare criterion and for any criterion that is increasing in the welfare of non bankers and that of bankers.

Our model does contain a number of extreme assumptions that deserve comment. In section 4, we assumed that there is no outside money. However, the results we obtained there can also be regarded as limiting results as the amount of indivisible outside money approaches zero. If outside money is indivisible, then the limit, as the amount of outside money approaches zero, of solutions to the optimum problem we posed is the solution with a zero amount of outside money.

There is a sense in which the assumed unit upper bound on individual holdings of notes or outside money forces us to assume that the amount of outside money is small. If there were no bound on individual holdings, then there is a sense in which any amount of indivisible outside money per type implies scarcity; the indivisibility 
implies that small enough transactions do not occur. ${ }^{11}$ Therefore, absent a bound on individual holdings, there would seem to be a role for note issue without any special assumptions about the amount of indivisible outside money.

The stationarity and symmetry we have imposed are responsible for, among other things, the finite set of steady state amounts of notes. We could avoid that consequence while maintaining stationarity and symmetry by having randomized strategies for bankers - for example, by having each banker issue a note to a nonbanker producer with some probability. We were reluctant to assume such randomness, because knowledge of trading histories would then have to be knowledge of the randomizing device being used. Another way to increase the set of steady state amounts of notes is to abandon symmetry and our extreme form of stationarity. For example, one could have a fraction of the bankers issue notes only at odd dates and the remainder issue notes only at even dates. That would permit smaller positive stocks of notes outstanding than does our assumption. Such possibilities matter because, as we saw in section 3, the optimal amount of money may be smaller than the smallest positive amount consistent with our specification..

The stationarity is also responsible for making our banks look quite different from historically observed note issuing banks. Our banks behave the same way no matter what happened to them in the past. That is, although our banks can be regarded as having balance sheets, the status of those balance sheets does not influence what they do. The analog of a balance sheet for one of our banks is a cumulative record of notes issued (liabilities) and notes destroyed (assets or credits). In our optimum, the implied net liability position, notes issued minus notes destroyed, is random and unbounded and does not affect what an individual banker does. A banker who has been lucky has a large deficit position (many more notes issued than destroyed) and one who been unlucky has a large surplus position (many more notes destroyed than issued). Such behavior is incentive feasible because we have assumed that there is perfect and costless knowledge of the trading histories of each banker. There are, no doubt, ways to weaken the assumed perfect knowledge of banker trading histories that would imply an optimum in which note issue by a banker is less likely the larger the banker's deficit position. Obviously, such a version would have banks that more closely resemble those we have observed.

\section{REFERENCES}

[1] Butlin, S. J., 1968. Foundations of the Australian Monetary System 1788-1851. (Sydney University Press, Sydney).

\footnotetext{
${ }^{11}$ Wallace and Zhou (forthcoming) study a model in which people are diverse in terms of their productivities and in which, therefore, there is an explicit role for transactions of different magnitudes.
} 
[2] Cavalcanti, R. de O., A. Erosa and T. Temzelides, 1996. Private money and reserve management in a matching model. Manuscript.

[3] Glassman, D. A., and A. Redish, 1988. Currency depreciation in early modern England and France. Explorations in Economic History 50, 789-805.

[4] Hanson, J. R. II, 1979. Money in the colonial American economy: an extension. Economic Inquiry 17, 281-286.

[5] Kocherlakota, N. and N. Wallace, 1977. Optimal allocations with incomplete record-keeping and no commitment. Working paper 578, Research Department. Federal Reserve Bank of Minneapolis.

[6] Law, J.,1705. Money and Trade.

[7] Shi, S., 1995. Money and prices: a model of search and bargaining. Journal of Economic Theory 67, 467-498.

[8] Trejos, A. and R. Wright, 1995. Search, bargaining, money and prices. Journal of Political Economy 103, 118-141.

[9] Wallace, N. and R. Zhou, forthcoming, A model of a currency shortage. Journal of Monetary Economics.

\section{APPENDIX}

\subsection{Proof of proposition 1.}

(A solution to Problem 2 exists. If $v^{*}(b)$ denotes the maximized value of $v$ in problem 2 and if $G(b)$ denotes the set of maximizers, then $v^{*}(b)$ is a continuous function and $G$ is $u h c$ in $b$.)

As noted, the boundedness of $u$ implies that the $v_{i}$ 's and $w$ are bounded and those bounds and the incentive constraints involving the production components of $s$ imply bounds on production. Let $v(b, s)$ and $\Gamma(b)$ denote, respectively, the values for the objective function and constraint set of problem 2 , associated with the parameter $b \in$ $\left(0, \frac{1}{N}\right]$ and coordinated action $s$. The bounds on production and the weak inequalities describing the feasibility conditions thus imply that $\Gamma(b)$ is compact. Since $v(b, \cdot)$ is clearly continuous, problem 2 describes the maximization of a continuous function over a compact set which thus has a solution. The proof is completed by showing that $\Gamma$ is continuous and by appealing to the Theorem of the Maximum. Notice that the constraint set depends on $b$ only because the $v_{i}$ 's and $w$ are functions of $b$. Since the $v_{i}$ 's and $w$ enter linearly in $\Gamma$ and are continuous in $b$, then $\Gamma$ is clearly continuous. 


\subsection{Proof of proposition 2 .}

(If $b \in\left(0, \frac{1}{N}\right)$, then any solution to problem 2 has bankers both creating and destroying notes.)

If $\max \{\alpha, \gamma, \theta\}=0$ or $\delta=0$, then, by (8)-(10), either $m_{1}=0$ and $\max \{\alpha, \gamma, \theta\}=0$ (no non banker has a note and none are being created), or $m_{0}=0$ and $\delta=0$ (every non banker has a note and none are being destroyed). Suppose that $s^{\prime}$ is incentive feasible and satisfies one of these conditions. It follows that $s^{\prime}$ is such that non bankers do not produce and that $v$ and $w$ are determined by two levels of output: production of bankers for bankers, $y^{\prime}$, and production of bankers for non bankers, denoted $z^{\prime}$. It follows that

$$
(1-\beta) v\left(s^{\prime}\right)=(1-\beta) v_{0}\left(s^{\prime}\right)=(1-\beta) v_{1}\left(s^{\prime}\right)=b u\left(z^{\prime}\right)
$$

and

$$
(1-\beta) w\left(s^{\prime}\right)=b\left[u\left(y^{\prime}\right)-y^{\prime}\right]-\left(\frac{1}{N}-b\right) z^{\prime} .
$$

We let $s^{\prime \prime}$ have $m_{0}^{\prime \prime}=m_{1}^{\prime \prime}=\frac{1}{2}\left(\frac{1}{N}-b\right) \equiv m, \gamma^{\prime \prime}=\theta^{\prime \prime}=0, \alpha^{\prime \prime}=\delta^{\prime \prime}=1, x_{n}^{\prime \prime}=x_{b}^{\prime \prime}=x$, $y^{\prime \prime}=y^{\prime}, y_{0}^{\prime \prime}=z^{\prime}$, and $y_{1}^{\prime \prime}=z^{\prime}+x$, for an $x$ to be determined. We show that there exist values of $x>0$ that imply incentive feasibility of $s^{\prime \prime}, v\left(s^{\prime \prime}\right)>v\left(s^{\prime}\right)$, and $w\left(s^{\prime \prime}\right)>w\left(s^{\prime}\right)$.

From (11) and (12), we have

$$
\begin{aligned}
(1-\beta)\left[m_{1}^{\prime \prime} v_{1}\left(s^{\prime \prime}\right)+m_{0}^{\prime \prime} v_{0}\left(s^{\prime \prime}\right)\right]= & m^{2}[u(x)-x]+m b u\left(z^{\prime}+x\right)+m b u\left(z^{\prime}\right)-m b x \geq \\
& m^{2}[u(x)-x]-m b x+2 m b u\left(z^{\prime}\right) .
\end{aligned}
$$

Therefore, to set the stage for the comparison between $v\left(s^{\prime \prime}\right)$ and $v\left(s^{\prime}\right)$, we have

$$
(1-\beta) v\left(s^{\prime \prime}\right) \geq \frac{1}{2}[m u(x)-(m+b) x]+b u\left(z^{\prime}\right)=\frac{1}{2}[m u(x)-(m+b) x]+(1-\beta) v\left(s^{\prime}\right) .
$$

From (11) and (12) we also have

$$
\left(\rho+\frac{1}{N}+b\right) \beta\left(v_{1}^{\prime \prime}-v_{0}^{\prime \prime}\right)=m[u(x)+x]+b\left[u\left(z^{\prime}+x\right)-u\left(z^{\prime}\right)+x\right],
$$

where $\rho=(1-\beta) / \beta$. Because $u\left(z^{\prime}+x\right)-u\left(z^{\prime}\right)>0$ for any $x>0$, it follows from (21) that $x \leq \beta\left(v_{1}^{\prime \prime}-v_{0}^{\prime \prime}\right)$ if

$$
\left(\rho+\frac{1}{N}+b\right) x \leq m[u(x)+x]+b x,
$$

or, equivalently, if 


$$
\frac{\rho+b+m}{m} x \leq u(x) .
$$

By the concavity of $u, u\left(z^{\prime}+x\right)-u\left(z^{\prime}\right) \leq u(x)$. Therefore, it follows from (21) that $u(x) \geq \beta\left(v_{1}^{\prime \prime}-v_{0}^{\prime \prime}\right)$ if $\left(\rho+\frac{1}{N}+b\right) u(x) \geq(m+b)[u(x)+x]$. This is implied by $(22)$.

Now we consider $w$. From (13), we have

$$
\begin{aligned}
(1-\beta) w\left(s^{\prime \prime}\right) & =b\left[u\left(y^{\prime}\right)-y^{\prime}\right]-m u(x)-m z^{\prime}-m\left(z^{\prime}+x\right) \\
& \left.=b\left[u\left(y^{\prime}\right)-y^{\prime}\right]-2 m z^{\prime}+m[u(x)-x)\right] \\
& =(1-\beta) w\left(s^{\prime}\right)+m[u(x)-x] .
\end{aligned}
$$

Because feasibility of $s^{\prime}$ implies $w\left(s^{\prime}\right) \geq 0$, (23) implies that the incentive constraint, $x \leq \beta w\left(s^{\prime \prime}\right)$, is satisfied provided

$$
\rho x \leq m[u(x)-x]
$$

which is equivalent to

$$
\left(1+\frac{\rho}{m}\right) x \leq u(x)
$$

Because $u^{\prime}(0)=\infty$, it is evident that there exists $x>0$ that satisfies the two incentive constraints, (22) and (24), and that also make the terms in $x$ on the far right sides of (20) and (23) positive. In particular, if we define $k$ by

$$
k=\max \left\{1+\frac{b}{m}, \frac{\rho+\frac{1}{N}-m}{m}, 1+\frac{\rho}{m}\right\}
$$

then $k>1$ and there exists a unique $x_{k}>0$ that satisfies $u\left(x_{k}\right)=k x_{k}$. Any $x \in\left(0, x_{k}\right)$ implies that $s^{\prime \prime}$ is incentive feasible and that $v\left(s^{\prime \prime}\right)>v\left(s^{\prime}\right)$ and $w\left(s^{\prime \prime}\right)>w\left(s^{\prime}\right)$.

\subsection{Proof of proposition 3.}

(Let $s^{*}(b)$ be a solution to problem 2 and let $v^{*}(b)$ be the maximized objective in that problem. If $b$ is sufficiently close to zero, then

(i) $s^{*}(b)$ satisfies $\alpha=\delta=1$ and $\gamma=\theta=0$ (which implies $m_{0}=m_{1} \equiv m$ );

(ii) if $x^{\prime}$ denotes the solution to $\frac{d u(x)}{d x}=1$, if $x^{\prime \prime \prime}$ denotes the unique positive $x$ satisfying $\left(1+\frac{\rho}{m}\right) x=u(x)$, and if $\hat{x}=\min \left\{x^{\prime}, x^{\prime \prime \prime}\right\}$, then $v^{*}(b) \rightarrow \frac{m}{2(1-\beta)}[u(\hat{x})-\hat{x}]$ as $b \rightarrow 0$; and

(iii) $v^{*}(b)$ is strictly increasing in $b$.) 
Proof of (i). We begin by maximizing $v$ without regard to the constraint $w \geq v$. From (11) and (12) and the definition of $v$,

$$
(1-\beta)\left(m_{0}+m_{1}\right) v=m_{0} m_{1}\left[u\left(x_{n}\right)-x_{n}\right]+b\left[m_{1} u\left(y_{1}\right)+m_{0} u\left(y_{0}\right)-m_{0} \alpha x_{b}\right] .
$$

We first show that the first term on the right side of $(25), m_{0} m_{1}\left[u\left(x_{n}\right)-x_{n}\right]$, is maximized at $m_{0}=m_{1}$ and that it is larger by a finite amount at $m_{1}=m_{0}$ than at any other feasible choice of $m_{1}$. From (11) and (12),

$$
\left[\rho+\frac{1}{N}-b+b \delta\left(1+\frac{m_{1}}{m_{0}}\right)\right] \beta\left(v_{1}-v_{0}\right)=m_{0} u\left(x_{n}\right)+m_{1} x_{n}+b\left[u\left(y_{1}\right)-u\left(y_{0}\right)+\alpha x_{n}\right],
$$

where, as before, $\rho=(1-\beta) / \beta$. Therefore, the incentive constraint, $x_{n} \leq \beta\left(v_{1}-v_{0}\right)$, is equivalent to

$$
\left[\rho+\frac{1}{N}-b+b \delta\left(1+\frac{m_{1}}{m_{0}}\right)\right] x_{n} \leq m_{0} u\left(x_{n}\right)+m_{1} x_{n}+b\left[u\left(y_{1}\right)-u\left(y_{0}\right)+\alpha x_{n}\right]
$$

Let $X\left(b ; y_{0}, y_{1}, \delta, \alpha\right)$ denote the set of non negative $x_{n}$ 's that satisfy $(27)$. This set is continuous in $b$ and because the production levels are bounded independently of $b$, $X\left(0 ; y_{0}, y_{1}, \delta, \alpha\right)$ is given by non negative $x_{n}$ 's that satisfy

$$
\left(\rho+\frac{1}{N}\right) x_{n} \leq m_{0} u\left(x_{n}\right)+m_{1} x_{n}
$$

or, equivalently,

$$
\left(1+\frac{\rho}{\frac{1}{N}-m_{1}}\right) x_{n} \leq u\left(x_{n}\right)
$$

Because $1+\rho /\left(\frac{1}{N}-m_{1}\right)$ is positive and increasing in $m_{1}$ for $m_{1} \in\left(0, \frac{1}{N}\right)$, and because $m_{1}=m_{0}$ is the smallest feasible and positive magnitude of $m_{1}$, it follows that for $b$ sufficiently close to zero, the set of $x_{n}$ 's satisfying (27) is larger at $m_{1}=m_{0}$ than at any other feasible and positive magnitude of $m_{1}$. It also follows, exactly as in section 3 , that if $x_{n}$ satisfies (28), then for sufficiently small $b$ it satisfies $u\left(x_{n}\right) \geq \beta\left(v_{1}-v_{0}\right)$. Thus, for $b$ sufficiently close to zero, the maximum of $u\left(x_{n}\right)-x_{n}$ is attained when $m_{1}=$ $m_{0}$. Moreover, the maximized value of $u\left(x_{n}\right)-x_{n}$ is positive. Also, it follows from the definition of $x^{\prime}$ and $x^{\prime \prime \prime}$ (see the statement of proposition 3) that the maximized value of $u\left(x_{n}\right)-x_{n}$ subject to $x_{n} \in X\left(b ; y_{0}, y_{1}, \delta, \alpha\right)$ approaches $u(\hat{x})-\hat{x}$ as $b \rightarrow 0$.

The maximum of $m_{0} m_{1}$ is uniquely attained at $m_{1}=m_{0}$ and the maximum is larger by a finite amount than at any other feasible choice of $m_{1}$. Therefore, for 
$b$ sufficiently close to zero, $m_{0} m_{1}\left[u\left(x_{n}\right)-x_{n}\right]$ is maximized at $m_{0}=m_{1}$ and the maximum is larger by a finite amount than at any other feasible choice of $m_{1}$. It follows that $m_{0}=m_{1}$ is necessary for maximization of $v$ when $b$ is sufficiently close to zero.

We now consider $w$. By (13), if $\alpha=0$, then $w \rightarrow 0$ as $b \rightarrow 0$. Therefore, $\alpha=1$ is necessary for attainment of a positive value of $w$ that is bounded away from zero as $b \rightarrow 0$.

We next show that it is incentive feasible to have $m_{0}=m_{1}, \alpha=1$, and $w \geq v$. We consider an allocation $s(b)$ given by $\alpha=\delta=1, \gamma=\theta=0, y_{0}=0$, and $y_{1}=x_{b}=x_{n}=y=x(b)$, where the function $x(b)$ is determined as now described. For such allocations, which imply $m_{0}=m_{1},(27)$ becomes

$$
\left(1+\frac{2 \rho}{\frac{1}{N}+b}\right) x \leq u(x) .
$$

Also, for such allocations, (26) implies that $u\left(x_{n}\right) \geq \beta\left(v_{1}-v_{0}\right)$ is equivalent to $u(x) \geq x(m+b) /(\rho+m+b)$, which is implied by (30). Therefore, we let $x^{\prime \prime}(b)$ be the unique positive solution for $x$ to (30) at equality, and let $x(b)=\min \left\{x^{\prime}, x^{\prime \prime}(b)\right\}$, where $x^{\prime}$ is the unique solution to $\frac{d u(x)}{d x}=1$. By construction, $s(b)$ satisfies the incentive constraints for non bankers. It remains to show that $s(b)$ satisfies the incentive constraints for bankers and that $w[s(b)] \geq v[s(b)]$. From (13),

$$
(1-\beta) w[s(b)]=\frac{\frac{1}{N}+b}{2}\{u[x(b)]-x(b)\},
$$

while from (25),

$$
(1-\beta) v[s(b)]=\frac{\frac{1}{N}+b}{4}\{u[x(b)]-x(b)\} .
$$

Therefore, $w[s(b)]>v[s(b)]$. The final step is to show that $x(b) \leq \beta w[s(b)]$. From (31), that inequality is the same as (30) and, therefore, is satisfied.

Proof of (ii). If we insert into (25) the necessary conditions for a maximum of $v$ when $b$ is sufficiently small and take the limit as $b \rightarrow 0$, the result is as asserted. Notice that the asserted limit is the limit of $v[s(b)]$ as given by (32). Because the limit of $v[s(b)]$ was obtained by maximizing $v$ without regard to the constraint that $w \geq v$ and because $s=s(b)$ implies $w \geq v$, it follows that $v^{*}(b) \rightarrow \lim _{b \rightarrow 0} v[s(b)]=$ $\frac{m}{2(1-\beta)}[u(\hat{x})-\hat{x}]$ as $b \rightarrow 0$.

Proof of (iii). Because the constraint set defined by (30) is increasing in $b$, it follows that $u[x(b)]-x(b)$ is weakly increasing in $b$. Therefore, by $(32), v[s(b)]$ is strictly increasing in $b$. Because $s(b)$ is incentive feasible for any $b,(31)$ and (32) are 
valid for any $b$. Therefore, for any $b, v[s(b)]$ is a lower bound on $v^{*}(b)$. The conclusion that $v^{*}(b)$ is strictly increasing in a neighborhood of $b=0$ follows from that bound, the continuity of $v^{*}(b)$, and the limit result in conclusion (ii). 